\title{
The Moving Plane Inhomogeneity Boundary with Transformation Strain
}

\author{
Xanthippi Markenscoff
}

Received: 1 December 2010 / Published online: 9 February 2011

(C) The Author(s) 2011. This article is published with open access at Springerlink.com

\begin{abstract}
Within the context of linear elastodynamics, the radiated fields (including inertia) for a plane inhomogeneous inclusion boundary with transformation strain (or eigenstrain), moving in general motion under applied loading, have been obtained on the basis of Eshelby's equivalent inclusion method, by using the strain field of a moving homogeneous inclusion boundary previously obtained. This dynamic strain field, obtained from the dynamic Green's function (for an isotropic material), is unique, and has as initial condition the limit of the spherical Eshelby inclusion, as the radius tends to infinity, which is the minimum energy solution for the half-space inclusion. With the equivalent dynamic eigenstrain (which is dependent on the velocity of the boundary), the radiated fields for the inhomogeneous plane inclusion boundary can be obtained, and from them the driving force on the moving boundary can be computed, consisting of a self-force (which is the rate of mechanical work (including inertia) required to create an incremental region of inhomogeneity with eigenstrain), and of a Peach-Koehler force associated with the external loading. While for an expanding plane homogeneous inclusion boundary the Peach-Koehler force is independent of the boundary velocity, in the case of an inhomogeneous one it is not.
\end{abstract}

Keywords Moving boundary $\cdot$ Inhomogeneity $\cdot$ Driving force $\cdot$ Transformation strain

Mathematics Subject Classification (2000) 74N20 · 74H05 · 74B99

\section{Introduction}

The analytic solution to the elastodynamic problem of a spherical dynamically expanding in general subsonic motion Eshelby inclusion with dilatational transformation strain, i.e., a spherically expanding boundary of strain discontinuity, in an isotropic material was obtained by Markenscoff and $\mathrm{Ni}$ [1] on the basis of a formula given by Willis [2] in terms of the dynamic Green's function. This solution is unique and has an initial condition the

X. Markenscoff $(\varangle)$

University of California, San Diego, La Jolla, CA 92093-0411, USA

e-mail: xmarkens@ucsd.edu 


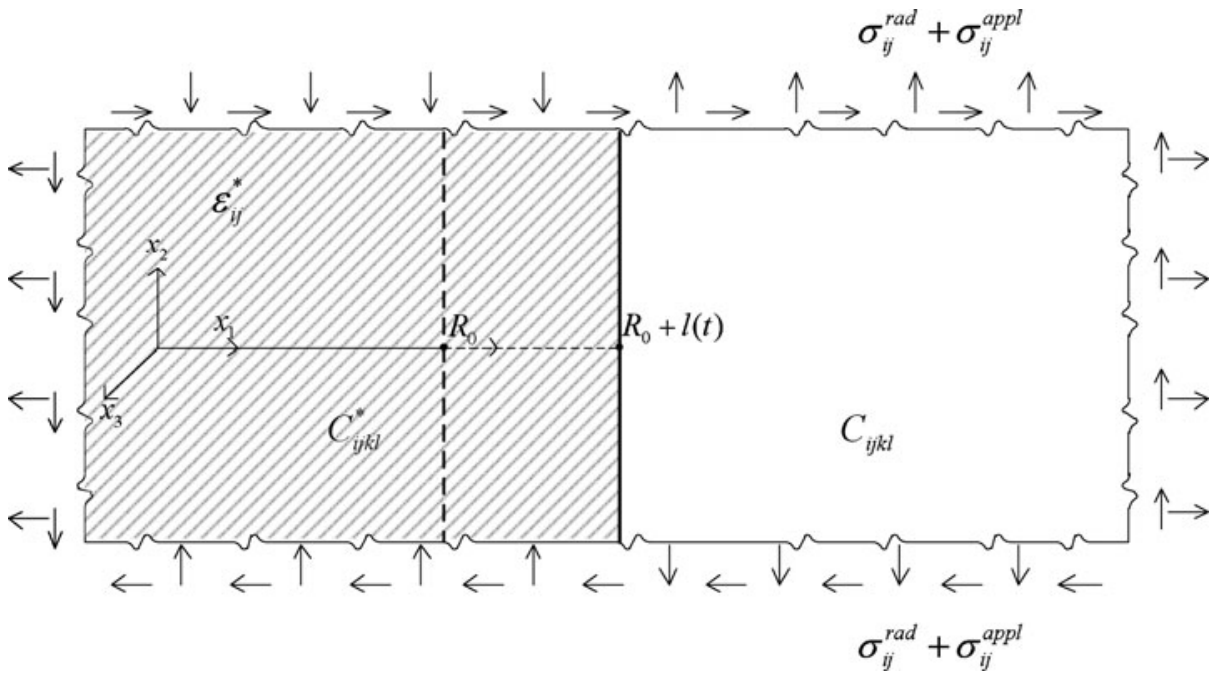

Fig. 1 The moving plane inhomogeneity boundary with transformation strain

static Eshelby [3] solution, which is a minimum energy solution. By a limiting procedure from the expanding spherical inclusion solution as the radius tends to infinity, the radiated fields of the plane moving constrained inclusion boundary were obtained. These are unique, and have as initial condition the limit of the static Eshelby solution, which is the minimum energy one for a half-space inclusion. The Hadamard jump conditions are satisfied on the moving surface of discontinuity, and time-dependent tractions are applied on the boundaries at infinity that are the limiting fields of the spherically expanding inclusion (see Fig. 1). Based on Willis' formula [2], the elastodynamic fields of a half-space constrained inclusion with general eigenstrain plane boundary moving from rest in general subsonic motion have also been obtained by Markenscoff and Ni [4]. This is also a unique solution with initial condition the minimum energy one.

Based on these radiated fields, the "driving force", obtained from the mechanical energyrelease rate which is the self-force on the moving boundary, was calculated by Markenscoff and Ni [1]. It has two parts: the static one, coinciding with Eshelby [5, 6] and Gavazza [7], and a dynamic one due to inertia, that depends on the velocity of the boundary. One may note, that the "driving force" expression of Atkinson and Eshelby [8], Freund [9] (for moving cracks) and Eshelby [5, 6] coincides for a purely mechanical system in an isothermal process with the expression for this force obtained by the phase transformation community (Abeyaratne [10], Truskinovsky [11], Heidug and Lehner [12], Abeyaratne and Knowles [13]) for a moving boundary of discontinuity. One may note that the "contour independent" dynamic $J$ integral (based on Noether's theorem), in the limit as it shrinks on the moving defect, is equivalent to the energy-release rate and the "driving force" Freund [14].

With the external loading included in the driving force expression, an additional term $\left\langle\sigma_{k \ell}^{a p p l}\right\rangle\left[\left[\varepsilon_{k \ell}^{*}(\mathbf{x}, t)\right]\right]$ is obtained which is the counterpart of the Peach-Kohler force on dislocations (Markenscoff and Ni [4]). Thus, the driving forces on moving defects, dislocations (Eshelby [15]) and inclusion boundaries, have exactly the same structure, a self-force due to inertia, and a Peach-Koehler force associated with the external loading. There is no coupling term of the applied loading with the inclusion boundary velocity. The self-force constitutes the mechanical rate of work needed to create an incremental region of eigenstrain by whatever source. Moreover, Gupta and Markenscoff [16] showed that the variation of the energy 
functional to be equal to the $J$ integral is a necessary and sufficient condition for equilibrium to be preserved in the domain during an incremental translation of the defect. The "Eshelby principle" (Eshelby [5]) that applying an external stress field (that renders the total driving force, or, equivalently, the total $J$ integral, equal to zero) can create mechanically an incremental region of eigenstrain has been demonstrated by Markenscoff [17] by a simple example.

In this paper, the analysis of Markenscoff and $\mathrm{Ni}$ [4] is extended to the plane constrained inhomogeneous (different elastic constants) inclusion moving boundary by extending the Eshelby method [3] of equivalent eigenstrain to dynamics. The equivalent eigenstrain is determined by the point-wise equivalence of the stresses, expressed in terms of strains by the constitutive relation. While for ellipsoidal inclusions, the total constrained strain is related to the equivalent eigenstrain by the Eshelby tensor, leading to a system of algebraic equations, in dynamics, it is related to the equivalent eigenstrain by the solution of Markenscoff and $\mathrm{Ni}$ [4]. As an example, the equivalent eigenstrain $\varepsilon_{i j}^{* *}$ is obtained analytically for a shear eigenstrain moving boundary and isotropic materials. Knowing the equivalent eigenstrain, the elastodynamic fields of a plane inhomogeneous inclusion moving boundary with transformation strain can be calculated from the Willis [2] formula, and, with these radiated fields, the driving forces can be obtained. Since the equivalent eigenstrain depends on the motion $l(t)$ of the boundary and the applied stress, the Peach-Koehler type force associated with the external loading now involves coupled terms of the loading with the motion and nonlinear terms in the applied stress.

Regarding applications, recently Yang, Escobar and Clifton [18], used a constrained Eshelby inclusion analysis to model the inducement of martensitic phase transformations from dynamically applied loading. The present analysis provides the inertia terms necessary for the modeling of this phenomenon, where, preliminary observations by Professor Rodney Clifton (of Brown University) confirm the behavior of the applied loading to boundary velocity relation obtained by Markenscoff and Ni [4], and Markenscoff [19], to predict the one of their experiments (private communication, R.J. Clifton, 2010).

\section{Radiated Fields from an Expanding Constrained Half-Space Inclusion with General Eigenstrain}

We present here briefly the formulation and the computed fundamental solution of Markenscoff and $\mathrm{Ni}$ [4] for an expanding plane constrained homogeneous inclusion boundary with general transformation strain, or equivalently called eigenstrain. It follows the analysis of Willis [2] treating constrained inclusions with time dependent eigenstrain, and, more specifically, (26) of Willis [2] for the displacement field $u_{i}(\mathbf{x}, t)$ due to domain and time dependent eigenstrain $\varepsilon_{i j}^{*}$ :

$$
u_{i}(\mathbf{x}, t)=\int_{-\infty}^{+\infty} d t^{\prime} \int_{D} C_{j k \ell m} \varepsilon_{\ell m}^{*}(\mathbf{x}, t) \frac{\partial}{\partial x_{k}} G_{i j}\left(\mathbf{x}-\mathbf{x}^{\prime}, t-t^{\prime}\right) d V^{\prime},
$$

where $D$ denotes the whole 3-dimensional space, $\varepsilon_{i j}^{*}$ the eigenstrain and $G_{i j}$ the dynamic Green's function for an impulse point force in an infinite medium, which for an isotropic material is

$$
G_{i j}\left(\mathbf{x}-\mathbf{x}^{\prime}, t-t^{\prime}\right)=g_{i j}\left(c_{2}\right)-g_{i j}\left(c_{1}\right)+\frac{\delta_{i j}}{c_{2}^{2} \bar{r}} \delta\left(\bar{t}-\frac{\bar{r}}{c_{2}}\right)
$$


where

$$
g_{i j}(c)=\left\{\frac{\bar{t}}{\bar{r}^{2}}\left(\frac{3 \bar{r}_{i} \bar{r}_{j}}{\bar{r}^{3}}-\frac{\delta_{i j}}{\bar{r}}\right) H\left(\frac{\bar{r}}{c}-\bar{t}\right)-\frac{\bar{r}_{i} \bar{r}_{j}}{\bar{r}^{3}} \frac{1}{c^{2}} \delta\left(\bar{t}-\frac{\bar{r}}{c}\right)\right\} \frac{1}{4 \pi \rho},
$$

with

$$
\begin{aligned}
& \bar{t}=t-t^{\prime}, \quad \bar{r}_{i}=x_{i}-x_{i}^{\prime}, \quad \bar{r}^{2}=\left(x_{1}-x_{1}^{\prime}\right)^{2}+\left(x_{2}-x_{2}^{\prime}\right)^{2}+\left(x_{3}-x_{3}^{\prime}\right)^{2}, \\
& c_{1}=\sqrt{(\lambda+2 \mu) / \rho}, \quad c_{2}=\sqrt{\mu / \rho} .
\end{aligned}
$$

We will apply (1) to a constrained inclusion occupying the half-space $x_{1} \leq R_{0}$ for $t \leq 0$ and expanding according to $x_{1}=R_{0}+\ell(t)$, such that $\ell(t)=0$ for $t \leq 0$, i.e.,

$$
\varepsilon_{\ell m}^{*}(\mathbf{x}, t)=\varepsilon_{\ell m}^{*} H\left(R_{0}+\ell(t)-x_{1}\right) .
$$

Like with dislocations moving from rest in general motion (Markenscoff [20] we will consider the solution of the problem with eigenstrain given by (3), to be the superposition of the two following problems, so that for Problem II boundary conditions of zero tractions at infinity apply:

Problem I Eigenstrain $\varepsilon_{i j}^{*} H\left(R_{0}-x_{1}\right)$ for $t \leq 0$, and corresponding displacement $u_{i}^{0}(x)$.

Problem II Eigenstrain $\varepsilon_{i j}^{*}\left[H\left(R_{0}+\ell(t)-x_{1}\right)-H\left(R_{0}-x_{1}\right)\right]$, and corresponding displacement denoted by $u_{i}^{*}$, and defined by:

$$
u_{i}^{*}(\mathbf{x}, t)=u_{i}(\mathbf{x}, t)-u_{i}^{0}(\mathbf{x}) .
$$

Regarding Problem I, in Markenscoff and Ni [1], the static half-space inclusion solution was obtained only for dilatational eigenstrain, while in Markenscoff and Ni [4] for general eigenstrain, as the limiting fields of the Eshelby static spherical inclusion (Mura [21], p. $68,(11.21) \mathrm{a})$, in the limit as the radius tends to infinity. The exterior fields of the halfspace inclusion are either obtained from the Eshelby exterior solution, or from the constant interior Eshelby one plus the Hill [22] jump conditions for the exterior. Equivalently, the exterior ones can also be obtained by considering the coupled system of equations expressing the continuity of tractions and compatibility of the deformation at the interface (see, also, Markenscoff [23]). They constitute the solution of Problem I.

It may be noted that the static solution for a half-plane inclusion was first obtained in 2D by Dundurs and Markenscoff [24] on the basis of a limiting procedure as the radius of a circular inclusion tends to infinity. This is the minimum energy solution for the half-space inclusion, since any superposed self-equilibrated and compatible tractions at infinity (called "rogue states" by Dundurs and Markenscoff [24]) increase the total energy of the system (e.g., Mura [21], p. 83, (13.8)).

We proceed with the solution of Problem II. Considering the fundamental equation (1), we have:

$$
\begin{aligned}
u_{i}^{*}(\mathbf{x}, t)= & \int_{-\infty}^{+\infty} d t^{\prime} \int_{-\infty}^{+\infty} d x_{1}^{\prime} d x_{2}^{\prime} d x_{3}^{\prime} C_{j k \ell m} \varepsilon_{\ell m}^{*}\left[H\left(R_{0}+\ell\left(t^{\prime}\right)-x_{1}^{\prime}\right)-H\left(R_{0}-x_{1}^{\prime}\right)\right] \\
& \times \frac{\partial}{\partial x_{k}^{\prime}} G_{i j}\left(\mathbf{x}-\mathbf{x}^{\prime}, t-t^{\prime}\right)
\end{aligned}
$$




$$
\begin{aligned}
= & \int_{0}^{\infty} d t^{\prime} \int_{-\infty}^{+\infty} d x_{1}^{\prime} d x_{2}^{\prime} d x_{3}^{\prime} C_{j k \ell m} \varepsilon_{\ell m}^{*}\left[\delta\left(R_{0}+\ell\left(t^{\prime}\right)-x_{1}^{\prime}\right)-\delta\left(R_{0}-x_{1}^{\prime}\right)\right] \\
& \times G_{i j}\left(\mathbf{x}-\mathbf{x}^{\prime}, t-t^{\prime}\right),
\end{aligned}
$$

since $G_{i j}=0$ at $x_{i}^{\prime}= \pm \infty, i=1,2,3$, and $\ell(t)=0$ for $t<0$.

Thus, the problem reduces to the evaluation of the integral in (5), namely

$$
\begin{aligned}
u_{i}^{*}(\mathbf{x}, t)= & \int_{0}^{\infty} d t^{\prime} \int_{-\infty}^{+\infty} d x_{1}^{\prime} C_{j i \ell m} \varepsilon_{\ell m}^{*}\left[\delta\left(R\left(t^{\prime}\right)-x_{1}^{\prime}\right)-\delta\left(R_{0}-x_{1}^{\prime}\right)\right] \\
& \times \int_{-\infty}^{+\infty} d \bar{r}_{2} d \bar{r}_{3} G_{i j}\left(\bar{r}_{1}, \bar{r}_{2}, \bar{r}_{3} ; \bar{t}\right)
\end{aligned}
$$

The evaluation is simplified by noting that the Green's function $G_{i j}\left(\bar{r}_{1}, \bar{r}_{2}, \bar{r}_{3} ; \bar{t}\right)$ is an odd function in $\bar{r}_{2}$ and $\bar{r}_{3}$ for $i \neq j$. Hence, the nonzero contributions to (9) are for $i=j$ only.

For an isotropic material the elastic coefficient tensor is

$$
C_{j k \ell m}=\lambda \delta_{j k} \delta_{\ell m}+\mu\left(\delta_{j \ell} \delta_{k m}+\delta_{j m} \delta_{k \ell}\right)
$$

so that

$$
C_{11 \ell m} \varepsilon_{\ell m}^{*}=C_{1111} \varepsilon_{11}^{*}+C_{1122} \varepsilon_{22}^{*}+C_{1133} \varepsilon_{33}^{*}=(\lambda+2 \mu) \varepsilon_{11}^{*}+\lambda\left(\varepsilon_{22}^{*}+\varepsilon_{33}^{*}\right)=A_{1}^{\prime},
$$

and (9) reduces, for $i=1$,

$$
\begin{aligned}
u_{1}^{*}(\mathbf{x}, t)= & \int_{0}^{\infty} d t^{\prime} \int_{-\infty}^{+\infty} d x_{1}^{\prime} A_{1}^{\prime}\left[\delta\left(R\left(t^{\prime}\right)-x_{1}^{\prime}\right)-\delta\left(R_{0}-x_{1}^{\prime}\right)\right] \\
& \times \int_{-\infty}^{+\infty} d \bar{r}_{2} d \bar{r}_{3} G_{11}\left(\bar{r}_{1}, \bar{r}_{2}, \bar{r}_{3} ; \bar{t}\right)
\end{aligned}
$$

where $R^{2}=\bar{r}_{1}^{2}+\bar{r}_{2}^{2}$, and according to the computations of Markenscoff and Ni [4],

$$
\int_{-\infty}^{+\infty} \int_{-\infty}^{+\infty} d \bar{r}_{2} d \bar{r}_{3} G_{11}\left(\bar{r}_{1}, \bar{r}_{2}, \bar{r}_{3} ; \bar{t}\right)=\frac{H\left(c_{1} \bar{t}-\left|\bar{r}_{1}\right|\right)}{2 \rho c_{1}}=\frac{c_{1} H\left(c_{1} \bar{t}-\left|x_{1}-x_{1}^{\prime}\right|\right)}{2(\lambda+2 \mu)} .
$$

Substituting (9) into (8) we have

$$
u_{1}^{*}(\mathbf{x}, t)=\int_{0}^{\infty} d t^{\prime} \frac{c_{1} A_{1}}{2}\left[H\left(\bar{t}-\frac{\left|x_{1}-R\left(t^{\prime}\right)\right|}{c_{1}}\right)-H\left(\bar{t}-\frac{\left|x_{1}-R_{0}\right|}{c_{1}}\right)\right]
$$

where

$$
A_{1}=\frac{A_{1}^{\prime}}{\lambda+2 \mu}=\left(\varepsilon_{11}^{*}+\frac{\lambda}{\lambda+2 \mu}\left(\varepsilon_{22}^{*}+\varepsilon_{33}^{*}\right)\right) .
$$

Moreover, it can be shown that

$$
\begin{aligned}
& \int_{0}^{\infty} d t^{\prime} H\left(t-t^{\prime}-\frac{\left|x_{1}-R_{0}\right|}{c_{1}}\right)=\left(t-\frac{\left|x_{1}-R_{0}\right|}{c_{1}}\right) H\left(t-\frac{\left|x_{1}-R_{0}\right|}{c_{1}}\right), \\
& \int_{0}^{\infty} d t^{\prime} H\left(t-t^{\prime}-\frac{\left|x_{1}-R_{0}-\ell\left(t^{\prime}\right)\right|}{c_{1}}\right)=\tau_{1} H\left(t-\frac{\left|x_{1}-R_{0}\right|}{c_{1}}\right)
\end{aligned}
$$


where $\tau_{1}, 0 \leq \tau \leq t$, is the unique solution of the equation

$$
f_{1}(\tau) \equiv c_{1}(t-\tau)-\left|x_{1}-R_{0}-\ell(\tau)\right|=0
$$

because the function $f_{1}(\tau)$ is monotonic for subsonic motion $|\dot{\ell}|<c_{1}$, and $f(0) \geq 0, f(t) \leq$ 0 , since it is solved only for $c_{1} t>\left|x_{1}-R_{0}\right|$.

From (14), (16) and (17), we have the solution for $u_{1}^{*}(\mathbf{x}, t)$

$$
u_{1}^{*}(\mathbf{x}, t)=\frac{c_{1} A_{1}}{2}\left[\tau_{1}-\left(t-\frac{\left|x_{1}-R_{0}\right|}{c_{1}}\right)\right] H\left(t-\frac{\left|x_{1}-R_{0}\right|}{c_{1}}\right) .
$$

Similarly, from (1),

$$
\begin{aligned}
u_{2}^{*}(\mathbf{x}, t)= & \int_{0}^{\infty} d t^{\prime} \int_{-\infty}^{+\infty} d x_{1}^{\prime} d x_{2}^{\prime} d x_{3}^{\prime} C_{j 1 \ell m} \varepsilon_{\ell m}^{*}\left[\delta\left(R\left(t^{\prime}\right)-x_{1}^{\prime}\right)-\delta\left(R_{0}-x_{1}^{\prime}\right)\right] \\
& \times G_{2 j}\left(\mathbf{x}-\mathbf{x}^{\prime}, t-t^{\prime}\right) \\
= & \int_{0}^{\infty} d t^{\prime} \int_{-\infty}^{+\infty} d x_{1}^{\prime} 2 \mu \varepsilon_{12}^{*}\left[\delta\left(R\left(t^{\prime}\right)-x_{1}^{\prime}\right)-\delta\left(R_{0}-x_{1}^{\prime}\right)\right] \\
& \times \int_{-\infty}^{+\infty} d \bar{r}_{2} d \bar{r}_{3} G_{22}\left(\overline{\mathbf{r}} ; t-t^{\prime}\right),
\end{aligned}
$$

and, according to Markenscoff and Ni [4], (16) reduces to the evaluation of the term

$$
\int_{-\infty}^{+\infty} d \bar{r}_{2} d \bar{r}_{3} G_{22}=\int_{-\infty}^{+\infty} d \bar{r}_{2} d \bar{r}_{3} \frac{\delta\left(\bar{t}-|\bar{r}| / c_{2}\right)}{c_{2}^{2} \bar{r} 4 \pi \rho}=\frac{H\left(c_{2} \bar{t}-\left|\bar{r}_{1}\right|\right)}{2 \rho c_{2}} .
$$

Substituting (20) into (21), we have

$$
\begin{aligned}
u_{2}^{*}(\mathbf{x}, t) & =\int_{0}^{\infty} d t^{\prime} \frac{2 \mu \varepsilon_{12}^{*}}{2 \rho c_{2}}\left[H-\left(\bar{t}-\frac{\left|x_{1}-R\left(t^{\prime}\right)\right|}{c_{2}}\right)-H\left(\bar{t}-\frac{\left|x_{1}-R_{0}\right|}{c_{2}}\right)\right] \\
& =\frac{c_{2} A_{2}}{2}\left[\tau_{2}-\left(t-\frac{\left|x_{1}-R_{0}\right|}{c_{2}}\right)\right] H\left(\bar{t}-\frac{\left|x_{1}-R_{0}\right|}{c_{2}}\right)
\end{aligned}
$$

where $A_{2}=2 \varepsilon_{12}^{*}$, and $\tau_{2}, 0 \leq \tau_{2} \leq t$, is the unique solution of the equation

$$
f_{2}(\tau)=c_{2}(t-\tau)-\left|x_{1}-R_{0}-\ell(\tau)\right|=0 \text { for subsonic motion }|\dot{\ell}|<c_{2} .
$$

In view of the symmetry between the $x_{2}$ and $x_{3}$ coordinates, we have

$$
u_{3}^{*}(\mathbf{x}, t)=\frac{c_{2} A_{3}}{2}\left[\tau_{2}-\left(t-\frac{\left|x_{1}-R_{0}\right|}{c_{2}}\right)\right] H\left(\bar{t}-\frac{\left|x_{1}-R_{0}\right|}{c_{2}}\right),
$$

where $A_{3}=2 \varepsilon_{13}^{*}$.

Thus (Markenscoff and Ni [4]), the solution for the displacement of the dynamic halfspace constrained expanding inclusion (superposition of Problem I plus Problem II):

$$
u_{1}(\mathbf{x}, t)=\frac{c_{1} A_{1}}{2}\left[\tau_{1}-\left(t-\frac{\left|x_{1}-R_{0}\right|}{c_{1}}\right)\right] H\left(t-\frac{\left|x_{1}-R_{0}\right|}{c_{1}}\right)+u_{1}^{0}(\mathbf{x}),
$$




$$
\begin{aligned}
& u_{2}(\mathbf{x}, t)=\frac{c_{2} A_{2}}{2}\left[\tau_{2}-\left(t-\frac{\left|x_{1}-R_{0}\right|}{c_{2}}\right)\right] H\left(t-\frac{\left|x_{1}-R_{0}\right|}{c_{2}}\right)+u_{2}^{0}(\mathbf{x}), \\
& u_{3}(\mathbf{x}, t)=\frac{c_{2} A_{3}}{2}\left[\tau_{2}-\left(t-\frac{\left|x_{1}-R_{0}\right|}{c_{2}}\right)\right] H\left(t-\frac{\left|x_{1}-R_{0}\right|}{c_{2}}\right)+u_{3}^{0}(\mathbf{x}),
\end{aligned}
$$

where

$$
\begin{aligned}
& A_{1}=\varepsilon_{11}^{*}+\frac{\lambda}{\lambda+2 \mu}\left(\varepsilon_{22}^{*}+\varepsilon_{33}^{*}\right), \quad A_{2}=\varepsilon_{12}^{*}+\varepsilon_{21}^{*}=2 \varepsilon_{12}^{*}, \\
& A_{3}=\varepsilon_{13}^{*}+\varepsilon_{31}^{*}=2 \varepsilon_{13}^{*}
\end{aligned}
$$

and, where $\tau_{i}$, with $0 \leq \tau_{i} \leq t$, is the unique solution for subsonic motion $|\dot{\ell}|<c_{2}$ of the equation

$$
c_{i}\left(t-\tau_{i}\right)=\left|x_{1}-R_{0}-\ell\left(\tau_{i}\right)\right|, \quad \text { for } i=1,2, \text { respectively, }
$$

and $u_{i}^{0}(\mathbf{x})$, for $i=1,2,3$, are the displacement solutions (modulo rigid body motion) for the static constrained half-space inclusion $\varepsilon_{i j}^{*} H\left(R_{0}-x_{1}\right)$.

\section{Propagating Plane Inclusion Boundary with Shear Eigenstrain}

Based on (25) to (27), we obtain the strain field for the dynamic half-space inclusion, either for motion with $\ell(t)>0$, or for motion with $\ell(t)<0$, including all possible subsonic motions with velocities of any sign, which would correspond to both expanding and shrinking motions (negative velocity for $\ell(t)>0$ ).

The total dynamic deformation (strain) field is obtained from the dynamic displacement solution

$$
\begin{aligned}
& \varepsilon_{11}=\frac{\partial u_{1}}{\partial x_{1}}=\frac{\partial u_{1}^{*}}{\partial x_{1}}+\frac{\partial u_{1}^{0}}{\partial x_{1}}, \quad \varepsilon_{22}=\frac{\partial u_{2}}{\partial x_{2}}, \quad \varepsilon_{33}=\frac{\partial u_{3}}{\partial x_{3}} \\
& \varepsilon_{12}=\frac{1}{2}\left(\frac{\partial u_{1}}{\partial x_{2}}+\frac{\partial u_{2}}{\partial x_{1}}\right)=\frac{1}{2}\left(\frac{\partial u_{1}^{0}}{\partial x_{2}}+\frac{\partial u_{2}^{*}}{\partial x_{1}}+\frac{\partial u_{2}^{0}}{\partial x_{1}}\right)=\frac{1}{2} \frac{\partial u_{2}^{*}}{\partial x_{1}}+\varepsilon_{12}^{0} \\
& \varepsilon_{13}=\frac{1}{2} \frac{\partial u_{3}^{*}}{\partial x_{1}}+\varepsilon_{13}^{0}, \quad \varepsilon_{23}=\varepsilon_{23}^{0},
\end{aligned}
$$

where $\varepsilon_{i j}^{0}$ for $i, j=1,2,3$, are the total strains field for the static half-space inclusion with the eigenstrain $\varepsilon_{i j}^{*} H\left(R_{0}-x_{1}\right)$, (Problem II).

If we are interested only in shear eigenstrain $\varepsilon_{12}^{*}$, then we have, for $c_{2} t>\left|x_{1}-R_{0}\right|$,

$$
\frac{\partial u_{2}^{*}}{\partial x_{1}}= \begin{cases}\frac{c_{2} A_{2}}{2}\left(\frac{\partial \tau_{2}}{\partial x_{1}}+\frac{1}{c_{2}}\right), & x_{1}>R_{0}, \\ \frac{c_{2} A_{2}}{2}\left(\frac{\partial \tau_{2}}{\partial x_{1}}-\frac{1}{c_{2}}\right), & x_{1}<R_{0} .\end{cases}
$$

In defining $\tau_{2}$, it can be proved (Markenscoff and $\mathrm{Ni}$ [4]) that, for the subsonic motion, i.e., $|\dot{\ell}|<c_{i}, x_{1}<R_{0}+\ell\left(\tau_{i}\right)$ if and only if $x_{1}<R_{0}+\ell(t)$, for $i=1,2$, respectively, so that (29) implies as in (Markenscoff and Ni [4])

$$
\frac{\partial \tau_{2}}{\partial x_{1}}= \begin{cases}\frac{1}{\dot{\ell}\left(\tau_{2}\right)-c_{2}}, & x_{1}>R_{0}+\ell(t), \\ \frac{1}{\dot{\ell}\left(\tau_{2}\right)+c_{2}}, & x_{1}<R_{0}+\ell(t) .\end{cases}
$$


For instance, the strain $\varepsilon_{12}$, which may be of particular interest, is

$$
\begin{aligned}
\varepsilon_{12}= & \varepsilon_{12}^{0}-\frac{1}{2}\left[\varepsilon_{12}^{*} \frac{\dot{\ell}\left(\tau_{2}\right)}{\dot{\ell}\left(\tau_{2}\right)+c_{2}} H\left(R(t)-x_{1}\right)-\varepsilon_{12}^{*} \frac{\dot{\ell}\left(\tau_{2}\right)}{\dot{\ell}\left(\tau_{2}\right)-c_{2}} H\left(x_{1}-R(t)\right)\right] \\
& \times H\left(t-\frac{\left|x_{1}-R_{0}\right|}{c_{2}}\right) .
\end{aligned}
$$

The stresses $\sigma_{12}$, as calculated by Markenscoff and $\mathrm{Ni}$ [4], are given below, as it should be clear that tractions must be applied at infinity to sustain this motion (see Fig. 1). With this time dependent loading on the boundaries at infinity, the solution constitutes the unique elastodynamic solution (with minimum energy initial condition) for the plane moving boundary of a constrained Eshelby inclusion.

$$
\begin{aligned}
\sigma_{12} & =2 \mu\left[\varepsilon_{12}-\varepsilon_{12}^{*} H\left(R(t)-x_{1}\right)\right] H\left(R_{0}-x_{1}\right) \\
& =2 \mu\left[\frac{1}{2}\left(\frac{\partial u_{1}}{\partial x_{2}}+\frac{\partial u_{2}}{\partial x_{1}}\right)-\varepsilon_{12}^{*} H\left(R(t)-x_{1}\right)\right],
\end{aligned}
$$

which, for both for expanding and shrinking motion, i.e., $\ell(t)>0$ and $\ell(t)<0$, is evaluated to be

$$
\begin{aligned}
\sigma_{12}= & \sigma_{12}^{0}-\left[\mu \varepsilon_{12}^{*} \frac{\dot{\ell}\left(\tau_{2}\right)}{\dot{\ell}\left(\tau_{2}\right)+c_{2}} H\left(R(t)-x_{1}\right)-\mu \varepsilon_{12}^{*} \frac{\dot{\ell}\left(\tau_{2}\right)}{\dot{\ell}\left(\tau_{2}\right)-c_{2}} H\left(x_{1}-R(t)\right)\right] \\
& \times H\left(t-\frac{\left|x_{1}-R_{0}\right|}{c_{2}}\right) .
\end{aligned}
$$

\section{Radiated Fields and Driving Forces for an Expanding Plane Boundary of an Inhomogeneity}

The radiated fields obtained by Markenscoff and Ni [4], as given by (25) to (27), can be used in order to obtain the radiated fields from the inhomogeneous (of different elastic constants $C_{i j k l}^{*}$ ) moving boundary. Eshelby's [3] equivalent inclusion method will be extended here to dynamics for a moving plane boundary of a half-space expanding inhomogeneity with transformation strain.

In statics, Eshelby [3] solved the inhomogeneity problem by reducing it to an inclusion problem (same elastic constants) with equivalent eigenstrain. Let the matrix have elastic constants $C_{i j k l}$ and the inhomogeneity $C_{i j k l}^{*}$, and consider that the inhomogeneity has eigenstrain $\varepsilon_{i j}^{*}$ and is under an applied loading at infinity $\sigma_{i j}^{a p p l}$ with corresponding strains $\varepsilon_{i j}^{a p p l}$ (satisfying $\sigma_{i j}^{a p p l}=C_{i j k l} \varepsilon_{k l}^{a p p l}$ ), and that the boundary moves according to $x_{1}=R_{0}+\ell(t)$ such that $\ell(t)=0$ for $t \leq 0$.

Then the total equivalent eigenstrain $\varepsilon_{i j}^{* *}$ is due to the inhomogeneity $\varepsilon_{i j}^{* i n h o m}$ (equivalent inclusion) plus the ("plastic") eigenstrain $\varepsilon_{i j}^{*}$ in the inclusion (i.e., Mura [21], (22.12a))

$$
\varepsilon_{i j}^{* *}=\varepsilon_{i j}^{* i n h o m}+\varepsilon_{i j}^{*} .
$$

The Eshelby equation that determines the eigenstrain $\varepsilon_{i j}^{* *}$ of the equivalent inclusion expresses the point-wise equivalence in the stresses (of the problem with the inhomogeneity and the equivalent to it inclusion), and is (e.g., Mura [21], (22.13a)):

$$
C_{i j m n} \varepsilon_{m n}^{* *}-\Delta C_{i j m n} \varepsilon_{m n}\left(\varepsilon_{k l}^{* *}\right)=C_{i j m n}^{*} \varepsilon_{m n}^{*}+\sigma_{i j}^{a p p l}-C_{i j m n}^{*} \varepsilon_{m n}^{a p p l},
$$


with

$$
\Delta C_{i j k l}=C_{i j k l}-C_{i j k l}^{*} \text {. }
$$

Equation (37), which expresses the equivalence in the stresses between inhomogeneity and inclusion, is valid for in statics and dynamics, if the total strain in (37) has the dynamic value that relates it to the eigenstrain. In the static case of the inclusion being an ellipsoid, the total strain $\varepsilon_{m n}$ in (37) is related to the equivalent eigenstrain by the Eshelby tensor

$$
\varepsilon_{m n}=S_{m n p q} \varepsilon_{p q}^{* *},
$$

and, inserted into (37), gives a system of linear algebraic equations for the equivalent eigenstrain $\varepsilon_{i j}^{* *}$.

In the case of an inhomogeneity of general shape $\Omega$, the total strain in (37) is obtained by the fundamental solution of an inclusion occupying the domain $\Omega$ in terms of the Green's function (e.g., Mura [21], (24.3))

$$
u_{m}(\mathbf{x})=-\int_{\Omega} G_{m k, l}\left(\mathbf{x}-\mathbf{x}^{\prime}\right) C_{k l r q} \varepsilon_{r q}^{* *}\left(\mathbf{x}^{\prime}\right) d \mathbf{x}^{\prime},
$$

so that, in view of (37) and (40), the equivalent eigenstrain in $\Omega$ is determined from the integro-differential equation (e.g., Mura [21], (24.4) with (24.5))

$$
\sigma_{i j}^{* *}(\mathbf{x})+\Delta C_{i j m n} \frac{\partial^{2}}{\partial x_{n} \partial x_{l}} \int_{\Omega} G_{k m}\left(\mathbf{x}-\mathbf{x}^{\prime}\right) \sigma_{k l}^{* *}\left(\mathbf{x}^{\prime}\right) d \mathbf{x}^{\prime}=q_{i j}(\mathbf{x}),
$$

where

$$
\sigma_{i j}^{* *}=C_{i j m n} \varepsilon_{m n}^{* *}, q_{i j}=\sigma_{i j}^{a p p l}-C_{i j m n}^{*} \varepsilon_{m n}^{a p p l} .
$$

In case of a dynamically expanding inhomogeneity with eigenstrain, in view of (1) and (37), the integro-differential equation (41) from which the equivalent eigenstrain may be determined, will now be written in terms of the dynamic Green's function as:

$$
\sigma_{i j}^{* *}(\mathbf{x}, t)+\Delta C_{i j m n} \frac{\partial^{2}}{\partial x_{n} \partial x_{l}} \int_{-\infty}^{\infty} d t^{\prime} \int_{\Omega(t)} G_{k m}\left(\mathbf{x}-\mathbf{x}^{\prime}, t-t^{\prime}\right) \sigma_{k l}^{* *}\left(\mathbf{x}^{\prime}, t^{\prime}\right) d \mathbf{x}^{\prime}=q_{0}(\mathbf{x}, t),
$$

where

$$
\sigma_{i j}^{* *}=C_{i j m n} \varepsilon_{m n}^{* *}, q_{i j}=\sigma_{i j}^{a p p l}-C_{i j m n}^{*} \varepsilon_{m n}^{a p p l} .
$$

In the case of the dynamically expanding plane boundary of an inhomogeneity with eigenstrain under applied loading, the integrations over the expanding inclusion domain can be performed for an isotropic material, and one can use the results of Markenscoff and Ni [4] of (25) to (27).

The point-wise equivalent stresses are given by the same equation

$$
C_{i j m n} \varepsilon_{m n}^{* *}-\Delta C_{i j m n} \varepsilon_{m n}\left(\mathbf{x}, t ; l(\tau), \varepsilon_{k l}^{* *}\right)=C_{i j m n}^{*} \varepsilon_{m n}^{*}+\sigma_{i j}^{a p p l}-C_{i j m n}^{*} \varepsilon_{m n}^{a p p l} \quad \text { in } R(t)>x_{1},
$$

with the total strain being $\varepsilon_{m n}\left(\mathbf{x}, t ; l\left(\tau_{i}\right), \varepsilon_{k l}^{* *}\right)$, where $\tau_{i}$ is the solution of

$$
c_{i}\left(t-\tau_{i}\right)-\left|x_{1}-R_{0}-l\left(\tau_{i}\right)\right|=0, \quad i=1,2,
$$

and is the time when a wavelet emitted by the moving front $l(\tau)$ has the time to reach the field point $(\mathbf{x}, t)$, and to contribute to the strain at that point, now related to the equivalent 
eigenstrain by the solution for the strain of the radiated fields of the moving inclusion plane boundary (with general eigenstrain), obtained above through (25), (26), (27), as a function of the eigenstrain and the boundary velocity.

With the total equivalent eigenstrain being determined from (37), the radiated fields for the moving boundary of an inhomogeneity with eigenstrain can be evaluated from (1):

$$
u_{i}(\mathbf{x}, t)=\int_{-\infty}^{+\infty} d t^{\prime} \int_{V(t)} C_{j k \ell m} \varepsilon_{\ell m}^{* *}\left(\mathbf{x}^{\prime}, t^{\prime} ; l\left(t^{\prime}\right)\right) \frac{\partial G_{i j}\left(\mathbf{x}-\mathbf{x}^{\prime}, t-t^{\prime}\right)}{\partial x_{k}} d V^{\prime},
$$

where $V(t)$ is the half-space inclusion with boundary moving according to $x_{1}=R_{0}+l(t)$.

As an example, we consider shear eigenstrain $\varepsilon_{12}^{*}$ and the inhomogeneity to be also an isotropic material (of different moduli than the matrix). Then, the strain $\varepsilon_{m n}\left(\mathbf{x}, t ; l(\tau), \varepsilon_{k l}^{* *}\right)$ is related to the eigenstrain from the radiated fields obtained above, where the eigenstrain will be now the total eigenstrain $\varepsilon_{i j}^{* *}$. In the case of shear eigenstrain only, the relationship is given in (33):

$$
\varepsilon_{12}=\varepsilon_{12}^{o}-\frac{1}{2} \varepsilon_{12}^{* *} \frac{\dot{\ell}\left(\tau_{2}\right)}{\dot{\ell}\left(\tau_{2}\right)+c_{2}} H\left(t-\frac{\left|x_{1}-R_{o}\right|}{c_{2}}\right), \quad \text { in } R(t)>x_{1},
$$

and, writing the static strain field in terms of the eigenstrain and the Eshelby tensor (Mura [21], (11.21)), (48) states

$$
\varepsilon_{12}=\frac{4-5 v}{15(1-v)} \varepsilon_{12}^{* *}-\frac{1}{2}\left(\frac{\dot{\ell}\left(\tau_{2}\right)}{\dot{\ell}\left(\tau_{2}\right)+c_{2}}\right) \varepsilon_{12}^{* *} H\left(t-\frac{\left|x_{1}-R_{o}\right|}{c_{2}}\right), \quad \text { in } R(t)>x_{1},
$$

where $\tau_{2}$ is the unique (for subsonic motion) solution of

$$
f_{2}\left(\tau_{2}\right)=c_{2}\left(t-\tau_{2}\right)-\left|x_{1}-R_{o}-\ell\left(\tau_{2}\right)\right|=0 .
$$

Solving from (45), the equivalent eigenstrain is

$$
\varepsilon_{12}^{* *}=\left\{2\left(\mu-\mu^{*}\right) \varepsilon_{12}^{a p p l}+2 \mu^{*} \varepsilon_{12}^{*}\right\} /\left\{4\left(\mu^{*}-\mu\right)\left[S_{1212}-\frac{1}{2} \frac{i\left(\tau_{2}\right)}{i\left(\tau_{2}\right)+c_{2}}\right]+2 \mu\right\},
$$

thus, extending the Eshelby equivalent inclusion formula [3, 25] (also, Mura [21] (22.19)) to dynamics, for a plane moving inhomogeneity boundary.

Expression (51) should enter into (47), according to which the radiated fields of an expanding inhomogeneity boundary in general subsonic motion $l(t)$ can be evaluated, most probably numerically. For a half-space inhomogeneity moving boundary, the radiated fields may be obtained, like for the inclusion moving boundary (Markenscoff and Ni [4]), by superposition of the static Problem I, which will be the Eshelby solution for the half-space inhomogeneity, as the limit of the spherical inhomogeneity as the radius tends to infinity, plus Problem II with the equivalent eigenstrain.

With those fields, that will depend on the motion $l(t)$ in a more complex way than in the case of the inclusion boundary (Markenscoff and $\mathrm{Ni}[1,4]$ ), the driving force per unit area of the inhomogeneity boundary, expressing the mechanical rate of work (per unit area) required to create an incremental volume of inhomogeneity with eigenstrain, will be computed from the mechanical energy-release rate (Atkinson and Eshelby [5], Freund [6]) on a contour surrounding the moving boundary and shrinking into it. For a moving discontinuity boundary, 
the energy-release-rate acquires the expression Abeyaratne [10] (also, see, Fried [26])

$$
f=[[W]]-\left\langle\sigma_{i j}\right\rangle\left[\left[\frac{\partial u_{i}}{\partial x_{j}}\right]\right],
$$

where the brackets denote jumps, and where we defined the symbol \langle\rangle by

$$
\langle A\rangle=\frac{1}{2}\left(A^{+}+A^{-}\right),
$$

and with the strain energy jump being

$$
[[W]]=\frac{1}{2}\left[\left[\sigma_{i j} \varepsilon_{i j}\right]\right]=\frac{1}{2} \sigma_{i j}\left(\frac{\partial u_{k}}{\partial x_{m}}-\varepsilon_{k m}^{* *}\right),
$$

with

$$
\sigma_{i j}=C_{i j k m}\left(\frac{\partial u_{k}}{\partial x_{m}}-\varepsilon_{k m}^{* *}\right) .
$$

Based on (54) and (55), the driving force expression of (52) reduces to (Markenscoff and $\mathrm{Ni}[1])$

$$
f=-\left\langle\sigma_{k m}\right\rangle\left[\left[\varepsilon_{k m}^{* *}(\mathbf{x}, t)\right]\right],
$$

and, in the case of an applied superposed loading at infinity, the total driving force will be

$$
f^{\text {self }}+\left\langle\sigma_{k \ell}^{a p p l .}\right\rangle \varepsilon_{k \ell}^{* *}(\mathbf{x}, t),
$$

with $f^{\text {self }}$ computed from the radiated field quantities obtained above through (47), and comprising the static Eshelby term [8] and the new inertia term.

From the structure of (57), we see that the Peach-Koehler type force term $\sigma^{a p p l} \varepsilon_{i j}^{*}$ (associated with the applied stress field) is not involving the velocity of the boundary in the case of a homogeneous inclusion moving boundary with constant eigenstrain $\varepsilon_{i j}^{*}$, but in the case of the inhomogeneously expanding boundary, since the equivalent eigenstrain $\varepsilon_{i j}^{* *}$ in (57) depends, through (45), both on the history of the boundary motion and the applied superposed stress, there will be coupling terms of the applied loading with the eigenstrain which involve the history of the motion of the boundary, as well as nonlinear terms in the applied stress.

It may be noted that the solutions for an expanding plane inclusion boundary obtained by Markenscoff and $\mathrm{Ni}$ [4] are valid and have the same expression for both expanding and shrinking subsonic motions. Moreover, if the boundary conditions on the boundaries $x_{2}=$ $\pm \infty, x_{3}= \pm \infty$ are traction-free, and not those of the limiting procedure (shown in Fig. 1), then the opposite loading may be applied to cancel the tractions, but these fields will not affect the fields in the bulk (see also, Dundurs and Markenscoff [24]), so that the driving force in the bulk will remain the same. It may be also noted that, while the elastodynamic solution for the moving plane inclusion boundary has been calculated analytically for an isotropic material, the solution for some classes of anisotropic materials may possibly be obtained partially analytically, as in the analysis of a generally moving dislocation in a cubic/hexagonal crystal by Markenscoff and Ni [27, 28], where the solution was obtained by Laplace transforms and the analysis of the Riemann surfaces corresponding to the four roots of the determinantal equation. Thus, the applicability to physical phenomena could be expanded. 
Open Access This article is distributed under the terms of the Creative Commons Attribution Noncommercial License which permits any noncommercial use, distribution, and reproduction in any medium, provided the original author(s) and source are credited.

\section{References}

1. Markenscoff, X., Ni, L.: The energy-release rate and self-force of dynamically expanding spherical and plane inclusion boundaries with dilatational eigenstrain. J. Mech. Phys. Solids 58, 1-11 (2010)

2. Willis, J.R.: Dislocations and Inclusions. J. Mech. Phys. Solids 13, 377-395 (1965)

3. Eshelby, J.D.: The determination of the elastic field of an ellipsoidal inclusion and related problems. Proc. R. Soc. Lond. A 241, 376-396 (1957)

4. Markenscoff, X., Ni, L.: Driving forces and radiated fields for expanding/shrinking half-space and strip inclusions with general eigenstrain. Quart. Appl. Math. (2010, in press)

5. Eshelby, J.D.: Energy relation of the energy-momentum tensor in continuum mechanics. In: Kanninen, M.F. et al. (eds.) Inelastic Behavior of Solids, pp. 77-115 (1970)

6. Eshelby, J.D.: Lectures on the elastic energy-momentum tensor (1977). In: Markenscoff, X., Gupta, A. (eds.) Collected Works of J.D. Eshelby: The Mechanics of Defects and Inhomogeneities, pp. 907-931. Springer, Dordrecht (2006)

7. Gavazza, S.D.: Forces on pure inclusion and Somigliana dislocations. Scr. Metall. 11, 979-981 (1977)

8. Atkinson, C., Eshelby, J.D.: The flow of energy into the tip of a moving crack. Int. J. Fract. Mech. 4, 3-8 (1968)

9. Freund, L.B.: Energy-flux into the tip of an extending crack in an elastic solid. J. Elast. 2, 341-349 (1972)

10. Abeyaratne, R.: An admissibility condition for equilibrium shocks in finite elasticity. J. Elast. 13, 175184 (1983)

11. Truskinovsky, L.: Equilibrium phase interfaces. Sov. Phys. Dokl. 27, 551-553 (1982)

12. Heidug, W.K., Lehner, F.K.: Thermodynamics of coherent phase transformation in nonhydrostatically stressed solids. Pure Appl. Geophys. 123, 91-98 (1985)

13. Abeyaratne, R., Knowles, J.K.: On the driving traction acting on a surface of strain discontinuity in a continuum. J. Mech. Phys. Solids 38, 345-36 (1990)

14. Freund, L.B.: Dynamic Fracture Mechanics. Cambridge University Press, Cambridge (1990)

15. Eshelby, J.D.: The equation of motion of a dislocation. Phys. Rev. 90, 248-255 (1953)

16. Gupta, A., Markenscoff, X.: Configurational forces as dissipative mechanism: a revisit. C. R., Méc. 336, 126-131 (2008)

17. Markenscoff, X.: Driving forces on phase boundaries: the Eshelby principle for an interface. Int. J. Fract. 165, 223-227 (2010)

18. Yang, S.-Y., Escobar, J., Clifton, R.J.: Computational modeling of stress wave induced martensitic transformations. Math. Mech. Solids 14, 220-257 (2009)

19. Markenscoff, X.: Evolution equation for moving defects: dislocations and inclusion boundaries. Int. J. Fract. 116, 35-40 (2010)

20. Markenscoff, X.: The transient motion of a nonuniformly moving dislocation. J. Elast. 10, $193-205$ (1980)

21. Mura, T.: Micromechanics of Defects in Solids. Martinus Nihjoff, The Hague (1982)

22. Hill, R.: Discontinuity relations in mechanics of solids. In: Sneddon, I.N., Hill, R. (eds.) Progress in Solid Mechanics. North-Holland, Amsterdam (1961)

23. Markenscoff, X.: On the shape of the Eshelby inclusions. J. Elast. 49, 163-166 (1998)

24. Dundurs, J., Markenscoff, X.: Stress fields and Eshelby forces on half-plane inhomogeneities and strip inclusions meeting a free surface. Int. J. Solids Struct. 46, 2481-2485 (2009)

25. Eshelby, J.D.: The continuum theory of lattice defects. Solid State Phys. 3, 79-144 (1956)

26. Fried, E.: Introduction. In: Ball, J.M., Kinderlehler, D., Podio-Guidugli, P. Slemrod, M. (eds.) Evolving Phase Interfaces in Solids: Fundamental Contributions to the Continuum Theory of Evolving Phase Interfaces in Solids, pp. 1-29. Springer, Berlin (1998)

27. Markenscoff, X., Ni, L.: Nonuniform motion of an edge dislocation in an anisotropic solid, I. Q. Appl. Math. 41, 475-494 (1984)

28. Markenscoff, X., Ni, L.: The transient motion of a dislocation in a solid of general anisotropy. J. Wave Motion 9, 191-197 (1987) 